\title{
INTERMITTENT 'SELF-LOCKING' OF GRAIN GROWTH IN FINE-GRAINED MATERIALS
}

\author{
Y. Estrin ${ }^{1}$, G. Gottstein ${ }^{2}$ and L.S. Shvindlerman ${ }^{2,3}$ \\ ${ }^{1}$ Department of Mechanical and Materials Engineering, The University of Western Australia, \\ Nedlands WA 6907, Australia ${ }^{2}$ Institut für Metallkunde und Metallphysik, RWTH Aachen, \\ Kopernikusstrasse 14, D-52074 Aachen, Germany ${ }^{3}$ Institute of Solid State Physics, \\ Russian Academy of Sciences, Chernogolovka, Moscow District 142432, Russia
}

(Received February 16, 1999)

(Accepted in revised form May 5, 1999)

Keywords: Grain growth; Nanocrystalline materials; Thermodynamic approach; Vacancy generation

\section{Introduction}

Grain boundary motion may be accompanied with vacancy generation [1-4]. A typical case where a moving grain boundary produces vacancies is in primary recrystallisation, when the growth of a recrystallised grain at the cost of a grain with a large dislocation density involves release of excess free volume. Vacancies are also generated during grain growth, when the total volume of the grain boundary 'phase', which is less dense than the bulk, decreases, again leading to release of excess free volume.

Interaction of moving grain boundaries with the vacancy atmospheres generated by themselves was shown to affect the grain boundary kinetics [2-4]. The effects are akin to impurity drag of grain boundaries. A kinetic approach taken in Refs. 2-4 made it possible to consider individual grain boundary motion. In the present communication, we look at the vacancy drag of grain boundaries during grain growth in a holistic way. Using a simple thermodynamic argument we shall determine conditions under which grain growth can be 'suffocated' by the concomitant vacancy generation. A simple criterion for 'self-locking' of grain growth will be presented. On this basis, a picture of intermittent grain growth is proposed in which stages of grain boundary motion are separated by periods of arrest. It is suggested that grain growth induced vacancy generation may be considered as a stabilising effect inhibiting grain growth in fine grained materials. The effect discussed is of particular significance for ultrafine grained polycrystals, notably nanocrystalline materials.

\section{Formulation of the Problem}

It is commonly believed that the main process occurring during grain growth is grain boundary migration leading to a reduction in the total grain boundary area. There is, however, another effect which always accompanies grain growth and which may have serious consequences. As is well known, the density of a grain boundary is lower than that of the bulk, see e.g. [5,6]. The excess free volume released during the reduction of the grain boundary 'phase' has to be accommodated by the bulk. It is natural to assume that this excess volume is assimilated by the bulk in the form of vacancies. This is 
supported by recent computer simulations of grain boundary motion [7]. The supply of these vacancies may increase the vacancy concentration in the material as compared with the equilibrium one, thus increasing the free energy of the system. This is tantamount to the rise of a thermodynamic resistance force. Under certain conditions, this effect may be as strong as to suppress grain growth altogether. In the following, the thermodynamic approach outlined above will be used to discuss the conditions for such 'self-locking' of grain growth.

The free energy of the system will be taken as the sum of the free energy of the grain boundaries,

$$
G_{G B}=\frac{3}{2} \cdot \frac{\sigma}{R}
$$

(where $\sigma$ is the free energy per unit area of grain boundary and $R$ is the average grain size), and that of the vacancies,

$$
G_{v}=G_{v}^{e q}+\frac{1}{2}\left(\frac{d^{2} G_{v}}{d c^{2}}\right)^{e q}\left(c-c^{e q}\right)^{2}
$$

considered not too far from equilibrium, so that a quadratic expansion about the equilibrium value can be used. Here $\mathrm{c}$ is the vacancy concentration, the superscript 'eq' referring to thermodynamic equilibrium. It should be noted that both contributions to the free energy are considered per unit volume. In obtaining eq. (1) spherical grains were assumed.

Using a text-book expression for the Gibbs free energy of $\mathrm{n}$ vacancies on $\mathrm{N}$ atomic sites per unit volume,

$$
G_{v}=n H_{f}-k T \ln \frac{N !}{(N-n) ! n !},
$$

where $H_{f}$ is the vacancy formation enthalpy, $T$ the absolute temperature and $k$ the Boltzmann constant, eq. (2) assumes the form

$$
G_{v}=G_{v}^{e q}+\frac{1}{2} \frac{N k T}{c^{e q}}\left(c-c^{e q}\right)^{2}
$$

Grain growth will obviously be permitted thermodynamically if the derivative of $G$ with respect to time $t$ is negative for positive $V=d R / d t$. In view of the above equations, this criterion reads

$$
\dot{G}=-\frac{3}{2} \frac{\sigma}{R^{2}} V+\frac{N k T}{c^{e q}}\left(c-c^{e q}\right) \dot{c}<0
$$

As will be seen below, we are interested in times when the absorption of vacancies by sinks is much slower than vacancy generation due to the loss of grain boundary specific area during grain growth. In other words, the time $t$ is considered to be much smaller than the characteristic time $\tau=d^{2} / D_{v}$ for vacancy removal to sinks. (Here $D_{v}$ is the vacancy diffusivity and $d$ the spacing between sinks, which can be identified with dislocations, grain boundaries themselves, or (particularly in thin films) the outer surface.) Under such conditions, the time derivative of the vacancy concentration is given by

$$
\dot{c}=\frac{6 \beta \delta}{R^{2}} V
$$


Here $\beta$ is the relative excess free volume of the boundary associated with a decreased density of a boundary, and $\delta$ is the boundary width; the factor of 6 corresponds to the particular (spherical) grain geometry considered.

Substitution of eq. (6) into inequality (5) yields

$$
-\frac{\sigma}{R^{2}} V+\frac{24 N k T}{c^{e q}}\left(\frac{\beta \delta V}{R^{2}}\right)^{2} t<0 .
$$

Evidently, this inequality can always be satisfied for sufficiently small $t$, implying that grain growth can occur initially. However, it is violated for

$$
t \geq t^{*}=\frac{1}{24} \cdot \frac{\sigma c^{e q} R^{2}}{N k T(\beta \delta)^{2} V}
$$

which means that grain growth gets arrested at $t=t^{*}$.

The assumption that $t^{*} \ll \tau=d^{2} / D_{v}$ made above can be rewritten as

$$
\frac{1}{24}\left(\frac{R}{d}\right)^{2} \frac{R}{N k T Z(\beta \delta)^{2}} \frac{D_{S D}}{m} \ll 1
$$

Here $D_{S D}=Z c^{e q} D_{v}=D_{o} \exp \left(-H_{S D} / k T\right)$ is the coefficient of bulk self-diffusion, $Z$ being the coordination number and $H_{S D}$ the corresponding activation enthalpy given by the sum of the activation enthalpy for vacancy migration (entering via $D_{v}$ ) and vacancy formation (entering via $c^{e q}$ ); the intrinsic grain boundary velocity $V$ has been replaced with $m \sigma / R$ where $m$ is the grain boundary mobility [8]. Inequality (9) is to be verified for each particular case of interest.

With the above results, the following scenario of grain growth can be suggested. Once started, grain growth will be arrested after a time $t^{*}$. Grain boundaries will remain stationary for a time $\tau-t^{*} \cong \tau$ during which time grain growth induced vacancy concentration will relax back to $c^{e q}$. The thermodynamic inhibition of grain growth will thus be removed and grain boundary motion can restart. The sequence of 'self-locking', relaxation of vacancy concentration in the bulk, and grain boundary motion will then be repeated cyclically. In other words, the motion of grain boundaries will occur in a jerky way. The intrinsic grain growth rate $V$, which in the absence of 'self-locking' would be determined by the driving force on the grain boundaries (that is, the capillary force), will be modified, so that the effective grain growth rate will be given by $V_{\text {eff }}=(t * / \tau) \cdot V$. This is the overall grain growth rate which would be observed in the experiment if the jerky character of grain boundary motion was not considered. The kinetics of grain growth and the attendant variation of the vacancy concentration are illustrated by a schematic diagram in Fig. 1.

The rough scenario outlined above is the more precise the smaller the ratio $t^{*} / \tau$. If $t^{*}$ is comparable with $\tau$, the jerky character of the grain boundary motion will be replaced with a continuous one. The condition expressing this situation reads

$$
\frac{1}{24} \cdot \frac{\sigma D_{S D}}{N k T Z(\beta \delta)^{2} V} \cdot\left(\frac{R}{d}\right)^{2} \cong 1 .
$$

Below the critical grain radius $R_{c}$ which can be obtained from eq. (10) the grain growth is affected by the self-locking described; above this grain size, the ordinary grain growth driven by capillary forces will prevail. As seen from eq. (10), the critical radius depends on the vacancy sink spacing $d$ which may be determined by the average distance between dislocations or the dislocation cell size. However, for ultrafine grain sized materials, where $d$ can be identified with $R$, the critical radius can be written as 

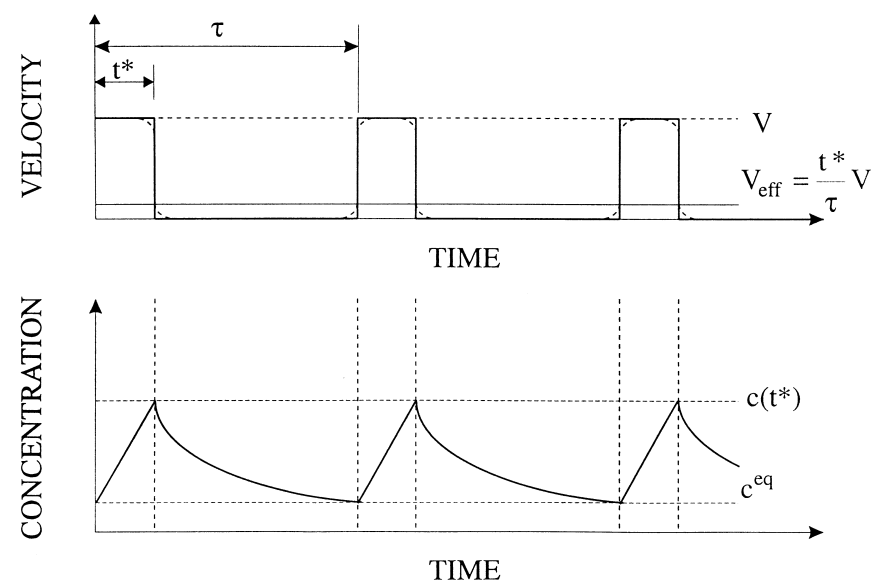

Figure 1. Time variation of (a) the grain boundary velocity and (b) the vacancy concentration (schematic). The dashed lines indicate-in an exaggerated way_-smoothening' of the predicted grain boundary velocity profile in a real situation.

$$
R_{c}=24 N k T Z(\beta \delta)^{2} \frac{m}{D_{S D}} .
$$

The above assumption that a vacancy generated in the process of grain growth in an ultrafine grained material has to migrate a distance of the order of the grain size implies that it does not have any affinity for the particular grain boundary in whose vicinity it has been generated.

It is interesting to note that in the jerky motion regime, the temperature dependence of the effective velocity $V_{\text {eff }}$ introduced above,

$$
V_{\text {eff }}=\frac{t^{*}}{\tau} \cdot V=\frac{1}{24} \cdot \frac{\sigma D_{S D}}{N k T Z(\beta \delta)^{2}} \cdot\left(\frac{R}{d}\right)^{2},
$$

is primarily determined by that of the coefficient of self-diffusion. This is a remarkable result, as it implies that different activation enthalpies should be observed for small and large grain sizes. Indeed, for small grain size where jerky motion prevails, the activation enthalpy for self-diffusion should be observed, while for large grain size corresponding to continuous grain boundary motion the activation enthalpy for the intrinsic grain boundary mobility will be found. The latter is generally different from the activation enthalpy for self-diffusion [8]. It should also be noted that as long as $R$ remains smaller than $R_{c}$, an acceleration of grain growth can be expected, as suggested by eq. (12) showing a quadratic dependence of the grain growth rate on $R$. However, on approaching the critical grain radius $R_{c}$, the 'normal' regime of grain growth driven by the surface tension will set in leading to the inverse $R$ dependence of the grain growth velocity.

Another interesting aspect of the grain growth kinetics relates to the early stages of the process in nanocrystalline materials. In this case, no vacancy sinks are available in the bulk of the grains due to the absence of dislocations there. The sink spacing $d$ is to be identified with the grain size $R$ yielding a time independent value of the average grain growth rate $V_{\text {eff }}$, cf. eq. (12),

$$
V_{e f f}=\frac{1}{24} \cdot \frac{\sigma D_{S D}}{N k T Z(\beta \delta)^{2}} .
$$

This indicates that in the initial stage of grain growth under the conditions considered above the grain size should be linear in time. 
TABLE 1

\begin{tabular}{lcr}
\hline $\mathrm{T}, \mathrm{K}$ & $V_{\text {eff }}^{\text {exp }}, \mathrm{ms}^{-1}$ & $V_{\text {eff, }}, \mathrm{ms}^{-1}$ \\
\hline 775 & $3.0 \times 10^{-12}$ & $1.2 \times 10^{-12}$ \\
805 & $1.2 \times 10^{-11}$ & $4.1 \times 10^{-12}$ \\
825 & $4.4 \times 10^{-11}$ & $2.0 \times 10^{-11}$ \\
\hline
\end{tabular}

\section{Experimental Examples}

Experimental data on the early stages of grain growth in nanocrystalline materials are fairly scarce. The available literature is referenced in a recent publication [9]. In that paper, grain growth in nanocrystalline iron was investigated in a range of temperatures. The above prediction of the linear initial grain growth can be checked against the data reported in Fig. 3 of Ref. 9. The data do support the predicted linearity of the initial parts of the grain size vs. annealing time diagrams. The data points for $775 \mathrm{~K}$, $805 \mathrm{~K}$ and $825 \mathrm{~K}$ appear suitable for a reasonably reliable determination of the initial linear slope. The values obtained (denoted $V_{e f f}^{\text {exp }}$ ) are compared with those calculated using eq. (13), i.e. $V_{\text {eff }}$ (Table 1). The coefficient of self-diffusion for Fe was taken from Ref. 10. A value of $0.8 \mathrm{Jm}^{-2}$ for $\sigma$ [11] was used.

The results of this comparison demonstrate a good agreement. It should be noted that notional values of the grain boundary parameters, $\beta=2 \times 10^{-2}$ and $\delta=10^{-9} \mathrm{~m}$, were taken in the above estimate. A slight adjustment of the product $\beta \delta$ would lead to a better numerical accord between the two columns of Table 1. However, even without this adjustment a correct temperature dependence of the measured grain growth rate is recovered by our calculations. The fact that this was achieved on the basis of self-diffusion data supports our prediction that for the regime when $t^{*}<\tau$ it is the coefficient of bulk self-diffusion which determines the temperature dependence of $V_{\text {eff }}$ The critical radius at which the linear time dependence of $R$ breaks down was also shown to be in agreement with the values estimated from the experimental curves in Ref. 9. A linear time dependence of the grain size also follows for the triple junction controlled kinetics [12]. In that case, the transition from the linear to the square root time dependence is associated with the change from the triple junction to the grain boundary kinetics.

A second experimental example which can be used to support the present approach is provided by the observations of grain growth in nanocrystalline $\mathrm{Ni}-1.2 \mathrm{wt} . \% \mathrm{P}$ with an initial grain size of 5 to $10 \mathrm{~nm}$ [13]. In situ measurements in a transmission electron microscope demonstrated that no grain growth occurred at $473 \mathrm{~K}$, while at $573 \mathrm{~K}$ and above discernible grain growth was recorded. Using the diffusion data for Ni from Ref. [14] we can estimate the two characteristic times, $t^{*}$ and $\tau$, for the two temperatures mentioned and the effective grain growth rate $V_{\text {eff }}$, eq. (12). While for $T=473 \mathrm{~K}, V_{\text {eff }}$ turns out to be several decades smaller than any discernible grain growth rate, for $T=573 \mathrm{~K}$ the measured value of about $5 \times 10^{-10} \mathrm{~cm} / \mathrm{s}$ follows from our estimate. The activation enthalpy for vacancy formation, $H_{f}$, which yields the observed velocity is $1.1 \mathrm{eV}$ : a value which is consistent with the data for pure $\mathrm{Ni}[15]$ and the notion that in the alloy under consideration it can be reduced by the amount of the binding energy between vacancies and $\mathrm{P}$ atoms.

\section{Conclusions}

The intent of this communication was to demonstrate that vacancy generation accompanying grain growth in fine grained materials has a strong inhibiting effect on the grain growth. Using a simple thermodynamic argument, it was shown that for sufficiently small grain sizes, the following features may be observed: 
(i) 'Self-locking' of grain growth when vacancy generation arrests grain boundary motion and intermittent 'stop and go' cycles (with arrest duration $t^{*}$ and period $\tau$ ) occur repetitively;

(ii) As a result of intermittent 'self-locking', jerky motion of the grain boundaries during grain growth is expected;

(iii) The kinetics of grain growth for the case of $t^{*}<\tau$ when 'self-locking' occurs are determined by bulk self-diffusion;

(iv) In nanocrystalline materials where sinks for vacancies are the grain boundaries themselves, a linear time dependence of the grain size follows; the corresponding grain growth rate and the critical radius up to which the linear dependence holds follow eqs. (13) and (11), respectively;

(v) A limited experimental evidence available supports the results obtained within the present approach.

\section{Acknowledgments}

Support from the Deutsche Forschungsgemeinschaft (DFG Grant 438 113/130/0) and from the International Office of the BMBF (Project AUS-032) is gratefully acknowleged. One of the authors (YE) would like to thank the Alexander von Humboldt Foundation for generous support through a Humboldt Research Award.

\section{References}

1. W. in der Schmitten, P. Haasen, and F. Häßner, Z. Metallk. 51, 101 (1960).

2. K. Lücke and G. Gottstein, Acta Metall. 29, 779 (1981).

3. Y. Estrin and K. Lücke, Acta Metall. 29, 791 (1981).

4. Y. Estrin, K. Lücke, and G. Gottstein, in Point Defects and Defect Interactions in Metals, Proceedings of the Fifth Yamada Conference, eds. J.-I. Takamura, M. Doyama, and M. Kiritani, p. 773, University of Tokyo Press, Tokyo (1982).

5. H. Gleiter, Acta Metall. 27, 187 (1979).

6. D. Wolf and K. L. Merkle, in Materials Interfaces, ed. D. Wolf and S. Yip, p. 87, Chapman \& Hall, London (1992).

7. M. Upmanyu, D. J. Srolovitz, G. Gottstein, and L. S. Shvindlerman, Interface Sci. 6, 289 (1998).

8. G. Gottstein, D. A. Molodov, and L. S. Shvindlerman, Interface Sci. 6, 7 (1998).

9. T. R. Malow and C. C. Koch, Acta Mater. 45, 2177 (1997).

10. G. Hettig, H. Mehrer, and K. Maier, Scripta Metall. 11, 795 (1977).

11. J. W. Taylor, J. Inst. Metals. 86, 456 (1957/58).

12. U. Czubayko, V. G. Sursaeva, G. Gottstein, and L. S. Shvindlerman, Acta Mater. 46, 5863 (1998).

13. K. Boylan, D. Ostrander, U. Erb, G. Palumbo, and K. T. Aust, Scipta Metall. Mater. 25, 2711 (1991).

14. H. Wollenberger, in Physical Metallurgy, 4th edn., ed. R. W. Cahn and P. Haasen, vol. 2, p. 1621, North-Holland, Amsterdam (1996).

15. B. S. Bokstein, Diffusion in Metals, Metallurgia Publishers, Moscow (1978). 